\title{
EL SOPORTE A LA INVESTIGACIÓN EN LAS BIBLIOTECAS UNIVERSITARIAS ESPAÑOLAS
}

\author{
Carina Rey Martín* \\ Facultad de Biblioteconomía y Documentación. Universidad de Barcelona \\ Enric Camón Luis ${ }^{* *}$ \\ Escuela Superior de Ciencias Sociales y de la Empresa. Tecnocampus. Universidad Pompeu Fabra. \\ Fernando Pacheco*** \\ Universidad Estatal de El Milagro. Ecuador.
}

\begin{abstract}
Resumen: En el contexto actual, en el que las universidades y su cuerpo docente deben adaptarse a los requerimientos legislativos vinculados al incremento de la investigación, las bibliotecas ocupan un rol crucial para dar respuesta a esta demanda, reforzando la prestación de servicios específicos de apoyo a los investigadores. El presente artículo pretende ofrecer una visión global de cuáles son los servicios de apoyo a la investigación prestados por las bibliotecas vinculadas a REBIUN. Con esta intención se llevó a cabo una encuesta dirigida a los responsables de las bibliotecas de dichas universidades. En este artículo se presentan y se analizan los datos recogidos, con especial incidencia en los servicios centrados en la formación, el asesoramiento y en la ayuda a la difusión de los resultados de investigación. Se concluye que algunos servicios de apoyo a la investigación se prestan de manera extendida desde las bibliotecas de las universidades, como por ejemplo los relacionados con el uso y formación de recursos de información. Sin embargo también es cierto que otros menos tradicionales, especialmente los vinculados a la integración de las bibliotecas en los proyectos de investigación, se prestan de manera poco homogénea entre las universidades analizadas. Este hecho podría tener respuesta en la necesidad de responder a las necesidades específicas de las políticas de investigación de los propios centros.
\end{abstract}

Palabras clave: servicios de apoyo a la investigación; bibliotecas universitarias; REBIUN; España.

Title: SUPPORT FOR RESEARCH OFFERED BY SPANISH UNIVERSITY LIBRARIES.

Abstract: In the current context, in which universities and their faculty must adapt their roll to legislative requirements linked to increased research, libraries play a crucial role in responding to this demand, reinforcing the provision of specific services to support Researchers. It is in this sense that the present article aims to provide an overview of which are the research support services provided by the libraries linked to REBIUN. With this intention, a survey was conducted among libraries's directors of these universities, and those results are offered in this paper. Thus, this article presents and analyzes the data collected, with a special focus on services focused on training, counseling and helping to disseminate the results of researchers. Regarding to principal findings, there is a global behavior among libraries in issues related to use and training on information resources. But there is a huge difference between libraries when the services to be offered are related to the integration of library services into researchers's team. This, perhaps, is due to the structure of the university where the library is acting.

Keywords: research support services; university libraries; academic libraries; REBIUN; Spain.

Copyright: (C) 2018 Servicio de Publicaciones de la Universidad de Murcia (Spain). Este es un artículo de acceso abierto distribuido bajo los términos de la licencia Creative Commons Reconocimiento 4.0 Internacional (CC BY 4.0).

\section{INTRODUCCIÓN}

Las bibliotecas universitarias españolas en los últimos años han adaptado sus estructuras y servicios en un entorno cambiante que requiere de capacidad de adaptación y vocación de servicio. En este sentido, y considerado lo mantenido por Gallo (2015), un nuevo modelo más vinculado a la gestión de la información está transformando globalmente a las bibliotecas. Desde sus infraestructuras hasta su denominación están en evolución con la finalidad de optimizar los recursos que ponen a disposición de sus comunidades académicas, tendiendo a la especialización en los servicios y productos de apoyo al aprendizaje, la docencia y la investigación. Concretamente, las universidades han centrado su atención en crear o mejorar el soporte a la investigación, alineándose con los requerimientos de los entes reguladores de la educación que las valoran y miden según su producción científica y los resultados de su actividad

\footnotetext{
* carina.rey@ub.edu

** ecamon@tecnocampus.cat

*** fernandopachecoolea@gmail.com
}

Recibido: 31-05-2017; $2^{\mathrm{a}}$ versión: 04-12-2017; aceptado: 15-12-2017.

REY MARTÍN, C., CAMÓN LUIS, E. y PACHECO, F. El soporte a la investigación en las bibliotecas universitarias españolas. Anales de Documentación, 2018, vol. 21, $\mathrm{n}^{\circ}$ 1. Disponible en: http://dx.doi.org/10.6018/analesdoc.21.1.295841. 
investigadora. Así pues, se aprecia la aparición de disposiciones legislativas y propuestas de entidades como el CSIC ${ }^{1}$ que promueven y apoyan la investigación y el desarrollo del proceso de creación científica. Sin embargo, no todas las universidades han apostado por el desarrollo de estos servicios de apoyo a la investigación desde la biblioteca.

En la literatura profesional de los últimos años se pueden encontrar numerosas referencias presentando las diversas tendencias que analizan cómo se produce este apoyo. Podemos destacar las aportaciones de Iribarren et al., (2015) donde se identifican los servicios de apoyo a la investigación que habitualmente se prestan desde las bibliotecas universitarias y el impacto que tienen en las necesidades del profesorado universitario. Por otro lado, existen otros referentes que se centran en cómo mejorar los servicios que ofrecen y que proponen nuevas formas de trabajar que amplían las potencialidades de los profesionales de las bibliotecas en el apoyo de la investigación. Un exponente de esta línea de trabajo es Shumaker (2012), con la propuesta de bibliotecarios incrustados o, más recientemente, Caridad (2013, p. 150), quien manifiesta que "El bibliotecario integrado ha de comprender la misión general y objetivos del grupo, su cultura de trabajo y formar parte del mismo".

Con anterioridad, en 2009, la prestigiosa entidad del ámbito bibliotecario OCLC desarrolló un estudio en el que establecía que era necesario fortalecer los servicios de soporte a la investigación y mejorar la interacción bibliotecarioinvestigador, basándose en experiencias recogidas en Australia, Países Bajos, Irlanda, Dinamarca y Reino Unido (OCLC, 2009). En este sentido, también pueden destacarse las aportaciones de autores australianos como Garner (2006) y Stokkem (2008). Concretamente, Garner apostaba ya entonces por la creación de espacios específicamente pensados para los investigadores dentro de las bibliotecas universitarias; mientras que Stokkem ponía en valor la creación de la figura de los gerentes de información. Cabe destacar que tener presente la particularidad de cada centro universitario y las necesidades de sus investigadores conduce a tal vez personalizar los servicios de apoyo a la investigación para dar respuesta a estas condiciones, tal y como estableció Rovira (2007).

Con estas adaptaciones de los servicios que se ofrecen podría pretenderse contar con bibliotecas fuertes que desempeñen un rol importante para lograr sociedades fuertes, considerando a Sipila (2015).

\section{OBJETIVOS Y METODOLOGÍA}

\subsection{Objetivos}

El objetivo de este artículo es analizar los servicios de apoyo a la investigación que las universidades españolas miembros de REBIUN prestan a través de sus bibliotecas. Si bien los servicios de apoyo a la docencia y al aprendizaje forman parte intrínseca de la cartera de servicios de las bibliotecas, los de apoyo a la investigación han ido tomando importancia en los últimos años. A pesar de ello, la prestación activa de estos no ha sido abordada de manera extensa en la literatura profesional, ni se dispone de muchos datos de uso.

Concretamente los objetivos son:

1. Determinar si las bibliotecas universitarias han abordado servicios de apoyo a la investigación.

2. Conocer qué servicios se prestan.

3. Analizar si la prestación se realiza exclusivamente por la biblioteca, o bien mediante la colaboración con otro servicio.

4. Identificar la modalidad en que se ofrecen, ya sea de manera presencial o virtual, o mediante la combinación de varias modalidades.

Por ello se dibuja una panorámica de cómo se ofrece el apoyo a la investigación en las bibliotecas de las universidades españolas miembros de REBIUN mediante un estudio empírico. Así pues, se desea constatar si, tal y como establece el III Plan estratégico de REBIUN 2020 mediante la línea estratégica II, se ofrecen servicios para "dar soporte a la docencia, aprendizaje, e investigación y gestión". Más allá del objetivo general de esta actuación estratégica de REBIUN, el concepto con el que se considera que debe proceder a la actuación es trabajando en la "personalización de nuevos servicios y espacios bibliotecarios y estableciendo marcos de colaboración e integración con otros servicios universitarios ${ }^{2,}$.

\subsection{Metodología}

Como instrumento metodológico se consideró que era adecuado el uso de un cuestionario dirigido a las direcciones de las bibliotecas universitarias asociadas a REBIUN. Este cuestionario debía poder realizarse vía web y por encima de todo debía garantizar el anonimato de las respuestas. Se pretendía obtener respuestas que dibujaran el panorama real de la prestación de los servicios, tomando en consideración el rigor profesional de las personas que respondían y 
garantizando el anonimato de las respuestas obtenidas. Así pues la finalidad era obtener respuestas reales y tangibles y no basadas en prospectivas de futuro.

\subsubsection{Diseño del cuestionario}

Para el diseño del cuestionario y de su contenido se tomaron en consideración distintos elementos. En primer lugar debía consignarse la estructura y el funcionamiento del propio cuestionario. Estos ítems fueron estructurados como una lista de servicios, los directores de las bibliotecas debían responder si formaban parte de sus catálogos. En segundo lugar, debía establecerse los elementos que formaban parte de esta lista de servicios y para ello se tomaron en consideración distintas aportaciones del ámbito profesional de las bibliotecas, de especial relevancia fueron las propuestas de estos servicios de Gannon-Leary; Bent; y Webb (2007), y de Torres-Salinas (2010). En tercer lugar era necesario también contextualizar el cuestionario a partir no solo de la literatura profesional mencionada previamente, sino también de las líneas de actuación que REBIUN incluye en sus planes estratégicos. En última instancia, y como fase previa al lanzamiento del cuestionario, se estableció una fase de pre-test. En esta se enriqueció el cuestionario con las apreciaciones de profesionales de las bibliotecas universitarias a quienes se consultó sobre la idoneidad del contenido y de la forma de presentación del cuestionario, y sobre las posibilidades técnicas de respuesta del mismo. Tomadas en consideración las recomendaciones derivadas de esta fase de pre-test, durante el segundo semestre del año 2016, se distribuyó vía web un cuestionario a los directores de las bibliotecas universitarias miembros de REBIUN ${ }^{3}$. El universo sobre el que se aplicó el cuestionario se dirigía a 76 bibliotecas. Si bien asociadas a REBIUN hay 77 bibliotecas, cabe considerar que de estas 76 son bibliotecas universitarias, y 1 es la Red de Bibliotecas del CSIC. En esta investigación se excluyó al CSIC al no tener la consideración formal de biblioteca universitaria puesto que no forma parte de la estructura de ninguna universidad.

\subsubsection{Contenido del cuestionario}

Para contextualizar el cuestionario se consideró necesario iniciar el mismo con una pregunta introductoria en el que se analizaba si las bibliotecas universitarias habían definido dentro de su cartera de servicios el apoyo a la investigación. Además se recogieron datos sobre aspectos formativos de los profesionales del servicio, y otros que puedan identificar la implicación y el compromiso de la biblioteca en la política de investigación del centro y en otros proyectos.

En lo concerniente al contenido de las preguntas específicas sobre el apoyo a la investigación, se establecieron cuatro ámbitos temáticos, los cuáles contenían el objeto de análisis. Estas cuatro agrupaciones daban respuesta a las necesidades básicas de los investigadores. El primer ámbito se correspondía con la disposición de un espacio físico específico dentro de las bibliotecas; el segundo analizaba la política de acceso a la información; el tercero se centraba en la evaluación y difusión de la producción científica; y en último lugar se preguntaba por el apoyo personalizado a los equipos de investigación.

\begin{tabular}{|c|c|}
\hline Tipo de servicio & Denominación \\
\hline Espacios físicos & Espacios de trabajo dirigidos a los investigadores \\
\hline Acceso a la información & $\begin{array}{l}\text { Servicio de obtención de documentos } \\
\text { Formación y utilización de bases de datos de alta especialización } \\
\text { Información bibliográfica especializada } \\
\text { Acceso a la información fuera del campus } \\
\text { Personal técnico especializado en las áreas de investigación de la institución }\end{array}$ \\
\hline $\begin{array}{l}\text { Evaluación y difusión de la } \\
\text { producción científica }\end{array}$ & $\begin{array}{l}\text { Formación y apoyo en la utilización de repositorios documentales } \\
\text { Formación y apoyo en la utilización de herramientas open access } \\
\text { Formación y apoyo en temas de derechos de autor } \\
\text { Gestión de currículum } \\
\text { Elaboración de índices bibliométricos } \\
\text { Asesoramiento sobre metodología de citación bibliográfica }\end{array}$ \\
\hline $\begin{array}{l}\text { Apoyo personalizado a los } \\
\text { equipos de investigación }\end{array}$ & $\begin{array}{l}\text { Gestión y mantenimiento de bibliografías } \\
\text { Gestión de comunicaciones entre el grupo } \\
\text { Preparación de borradores } \\
\text { Asesoramiento sobre las revistas donde publicar }\end{array}$ \\
\hline
\end{tabular}

Tabla I.- Catálogo de servicios de apoyo a la investigación. (Elaboración propia).

Para poder ofrecer un análisis más detallado se consideró que conocer desde qué servicio se prestaban podría aportar indicadores complementarios. En este sentido, se estableció que la prestación de estos podía responder a cuatro opciones según fuera: 
- responsabilidad de la biblioteca exclusivamente

- responsabilidad de la biblioteca conjuntamente con otro servicio

- responsabilidad directa de otro servicio, sin participación de la biblioteca

- un servicio que no se prestaba en la universidad

Con estas consideraciones relacionadas con la prestación de los servicios, se pretendía dar respuesta a lo establecido por REBIUN en la línea estratégica II cuándo menciona la necesidad de establecer "marcos de colaboración e integración con otros servicios universitarios".

\section{RESULTADOS}

Después del periodo de recogida de información, que concluyó en noviembre de 2016, se procedió al análisis de los resultados. Se obtuvieron 42 respuestas que supone un 55\% de la totalidad de los encuestados del universo posible. En términos de titularidad, participaron 32 universidades públicas y 10 privadas. Se ha considerado necesario realizar esta diferenciación sobre la titularidad de las universidades que participaron en la respuesta del cuestionario por si este elemento podría ser el origen de diferencias significativas vinculadas a la titularidad del centro. En términos porcentuales, se dispone de datos que representan al $64 \%$ de bibliotecas de universidades de titularidad pública, y al $38 \%$ de las de titularidad privada.

Los resultados analizados consideran su impacto respecto de las respuestas obtenidas y no respecto del universo posible, puesto que al no disponer de datos de todas las instituciones no pueden extrapolarse los resultados al conjunto de todas las bibliotecas universitarias.

\subsection{Identificación del servicio de apoyo a la investigación}

Un objetivo específico de este trabajo es determinar la identificación en el funcionamiento de los sistemas del servicio de apoyo a la investigación. Para ello el cuestionario contenía una pregunta específica para este ítem. Las respuestas indican que 36 universidades tienen definido un servicio de apoyo a la investigación, es decir un $85 \%$ del total de universidades que han respondido ofrecen este servicio. Analizando la prestación de manera más específica, se detecta que es así en un $90 \%$ en el caso de las de titularidad pública, y un $75 \%$ en las privadas, la implementación del apoyo a la investigación comporta algunos cambios o modificaciones en la organización, una de ellas es su inclusión dentro de los organigramas. De los centros que han participado en el estudio, un $85,6 \%$ han realizado esta modificación del organigrama del servicio bibliotecario.

Sin embargo, cuando se analizan las respuestas a la prestación efectiva de los ítems que se identifican dentro de la cartera de servicios vinculados a este apoyo, se obtienen respuestas superiores por lo que puede darse el caso que no se haya formalizado el servicio como tal en la biblioteca pero que, a pesar de ello, se esté prestando.

\subsection{Catálogo de servicios de apoyo a la investigación}

El segundo de los objetivos era específicamente conocer como es la prestación de los servicios de apoyo a la investigación exclusivamente por parte de la biblioteca.

Los datos recogidos se presentan en formato de valor absoluto y porcentaje relativo a la muestra analizada para el conjunto de las bibliotecas universitarias, y después se disgregan entre la titularidad de la institución. 


\begin{tabular}{|c|c|c|c|c|c|c|}
\hline \multirow[b]{2}{*}{ Denominación del servicio } & \multicolumn{2}{|c|}{ Total bibliotecas } & \multicolumn{2}{|c|}{ Titularidad pública } & \multicolumn{2}{|c|}{ Titularidad privada } \\
\hline & Valor & $\%$ & Valor & $\%$ & Valor & $\%$ \\
\hline $\begin{array}{l}\text { Espacios de trabajo dirigidos a los } \\
\text { investigadores }\end{array}$ & 32 & $76 \%$ & 21 & $70 \%$ & 11 & $92 \%$ \\
\hline $\begin{array}{l}\text { Servicio de obtención de } \\
\text { documentos }\end{array}$ & 41 & $98 \%$ & 29 & $97 \%$ & 12 & $100 \%$ \\
\hline $\begin{array}{l}\text { Formación y utilización de bases } \\
\text { de datos de alta especialización }\end{array}$ & 42 & $100 \%$ & 30 & $100 \%$ & 12 & $100 \%$ \\
\hline $\begin{array}{l}\text { Información } \\
\text { especializada }\end{array}$ & 40 & $95 \%$ & 28 & $93 \%$ & 12 & $100 \%$ \\
\hline $\begin{array}{l}\text { Acceso a la información fuera del } \\
\text { campus }\end{array}$ & 32 & $76 \%$ & 23 & $77 \%$ & 9 & $75 \%$ \\
\hline $\begin{array}{l}\text { Personal técnico especializado en } \\
\text { las áreas de investigación de la } \\
\text { institución }\end{array}$ & 32 & $76 \%$ & 24 & $80 \%$ & 8 & $67 \%$ \\
\hline 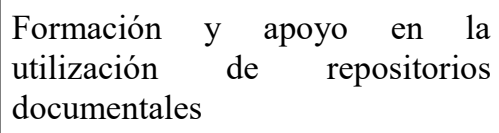 & 41 & $98 \%$ & 30 & $100 \%$ & 11 & $92 \%$ \\
\hline $\begin{array}{l}\text { Formación y apoyo en la } \\
\text { utilización de herramientas open } \\
\text { access }\end{array}$ & 38 & $90 \%$ & 28 & $93 \%$ & 10 & $83 \%$ \\
\hline $\begin{array}{l}\text { Formación y apoyo en temas de } \\
\text { derechos de autor }\end{array}$ & 36 & $86 \%$ & 27 & $90 \%$ & 9 & $75 \%$ \\
\hline Gestión de currículums & 8 & $19 \%$ & 7 & $23 \%$ & 1 & $8 \%$ \\
\hline $\begin{array}{l}\text { Elaboración de índices } \\
\text { bibliométricos }\end{array}$ & 26 & $62 \%$ & 17 & $57 \%$ & 9 & $75 \%$ \\
\hline $\begin{array}{l}\text { Asesoramiento sobre metodología } \\
\text { de citación Bibliográfica }\end{array}$ & 42 & $100 \%$ & 30 & $100 \%$ & 12 & $100 \%$ \\
\hline $\begin{array}{l}\text { Gestión y mantenimiento de } \\
\text { bibliografías }\end{array}$ & 29 & $69 \%$ & 22 & $73 \%$ & 7 & $58 \%$ \\
\hline $\begin{array}{l}\text { Gestión de comunicaciones entre } \\
\text { el grupo }\end{array}$ & 4 & $10 \%$ & 3 & $10 \%$ & 1 & $8 \%$ \\
\hline Preparación de borradores & 1 & $2 \%$ & 1 & $3 \%$ & 0 & $0 \%$ \\
\hline $\begin{array}{l}\text { Asesoramiento sobre las revistas } \\
\text { donde publicar }\end{array}$ & 32 & $76 \%$ & 24 & $80 \%$ & 8 & $67 \%$ \\
\hline
\end{tabular}

Tabla II.- Índice de prestación de los servicios de apoyo a la investigación ofrecidos por la biblioteca. (Elaboración propia).

Cabe destacar que en este apartado, y considerando de nuevo el Plan estratégico de REBIUN cuando menciona la necesidad de cooperar con otros servicios, el cuestionario se diseñó para que las bibliotecas analizadas respondieran sobre si la prestación de servicios de apoyo a la investigación, es una actividad compartida conjuntamente con otro servicio. Estos son los resultados de esta cooperación entre estamentos de las universidades. 
Además se preguntó si los servicios de apoyo a la investigación analizados, eran directamente ofrecidos por otros servicios, o bien no se ofrecían en el conjunto de la universidad. Pudiéndose obtener una visión global de la prestación del apoyo a la investigación por parte de las universidades. Los datos relacionados con esta última opción se destacan cuando resultan relevantes dentro de los epígrafes que valoran específicamente cada servicio.

Para facilitar el análisis de los datos, se organizan en tres bloques considerando la intensidad en la prestación de los resultados.

\subsubsection{Análisis de los resultados}

Los resultados presentan los servicios que se ofrecen de manera más extendida por parte de la biblioteca en primera instancia. Así pues se ordenan de mayor a menor índice de prestación, y se clasifican entre aquellos que dependen de la biblioteca para el conjunto de las universidades y después aquellos que a pesar de ofrecerse de manera muy significativa desde esta, precisan de la colaboración de otros estamentos de la universidad en cuestión.

\subsubsection{Servicios prestados de manera intensiva y exclusivamente por la biblioteca}

Del análisis de los datos se destacan una serie de servicios que forman parte del cuerpo central de prestación de las bibliotecas universitarias y que, aparentemente, no precisan de políticas activas de promoción de los mismos.

Aspectos que apoyan el eje central de la investigación como "la formación y la utilización de bases de datos de alta especialización y el asesoramiento sobre metodologías de citación bibliográfica”, se ofrecen en todas las universidades, con un $100 \%$ de respuesta, sin diferenciarse entre el valor obtenido entre la titularidad de la institución.

Le siguen la "obtención de documentos", prestado por 41 de las 42 bibliotecas que contestaron lo que equivale a un $98 \%$. De este porcentaje global, un $97 \%$ de públicas y un $100 \%$ de privadas y la "información bibliográfica especializada", con un $95,2 \%$ de respuestas en global. En términos de titularidad, en el ámbito privado la totalidad lo presta, mientras que este valor llega al $93 \%$ en el caso de las públicas. En relación con estos dos servicios, cabe destacar que en el caso de las públicas, una universidad afirmó que se prestaba por otro servicio ajeno a la biblioteca, sin indicar cuál.

La "formación y apoyo en la utilización de repositorios documentales" es ofrecida por el 98\% de las bibliotecas, es decir 41 de 42 . El 100\% en las universidades públicas lo ofrecen, mientras el 92\% lo presta desde las bibliotecas en las universidades privadas.

Los cuatro elementos considerados en este bloque de información forman parte tradicionalmente en la cartera de servicios de las bibliotecas universitarias. Al que se añade como elemento propio la formación y apoyo en la utilización de repositorios documentales.

\subsubsection{Servicios prestados por la biblioteca y otro servicio de la universidad}

Seguidamente se analizan aquellas prestaciones que si bien se prestan por parte de la biblioteca al menos en un $60 \%$ de los casos. Además se identifica y se realiza mediante la concurrencia de la biblioteca y otro servicio.

Los "espacios de trabajo dirigidos específicamente a los investigadores" se ofrecen en un 76,2\% de los casos desde la biblioteca, en esta ocasión en mayor medida entre las privadas (92\%) que las públicas (70\%). A título de comentario, cabe destacar que en una universidad pública se ofrece conjuntamente con otro servicio en un caso, y en otra directamente por otro servicio. Ello supone que las autoridades universitarias han reformulado los espacios habituales de la biblioteca para ofrecer este espacio diferenciado y que han dispuesto de una aportación económica para realizarlo.

La "formación y apoyo en la utilización de herramientas de open access $(O A)$ " se ofrece en un 90,5\% de las bibliotecas analizadas. Hay que destacar que este servicio se administra conjuntamente con otros servicios de la universidad en un 7,1\% de las bibliotecas participantes, específicamente en un 3\% en las bibliotecas de universidades públicas, porcentaje que se sitúa en el $17 \%$ en las privadas. Se observa que este servicio que no categorizaríamos como tradicional, se ofrece en un porcentaje alto de servicios bibliotecarios, lo que denota que la filosofía open access está incorporándose a la práctica bibliotecaria.

Respecto de la "formación y apoyo en temas de derechos de autor" se han obtenido un 85,7\% de respuestas afirmativas, tanto en públicas como en privadas. De estas, un 9,5\% (distribuidas de igual forma en valor absoluto entre públicas y privadas) indican que se realiza conjuntamente con otro servicio y un $2,4 \%$ que corresponde a una 
universidad privada, que lo ofrece otro servicio. Es por tanto un servicio que tiene una aceptación muy amplia en el ámbito bibliotecario universitario.

El "acceso a la información fuera del campus" se presta en 32 de las $42(76,2 \%)$ instituciones tanto de titularidad pública (77\%) como privadas (75\%). Más concretamente en un 14,2\% se ofrece conjuntamente con otros servicio de la universidad (13\% en públicas y $17 \%$ en privadas) y un $2,4 \%$ indican que es un servicio que no corresponde a su competencia. Este hecho bien podría deberse a la necesidad de contar con soluciones técnicas y tecnológicas, más vinculadas a los servicios informáticos, que no dependen exclusivamente de la biblioteca que es la encargada de gestionar los recursos de información.

En lo concerniente a si se dispone de "personal técnico especializado temáticamente en las áreas de investigación de la institución", se ha obtenido una respuesta afirmativa de un 76,2\%. 24 bibliotecas de titularidad pública lo prestan directamente y en 1 caso lo hacen con otro servicio, mientras que en el ámbito privado, 8 lo prestan y afirman a su vez que en una ocasión se realiza desde otro estamento distinto a la biblioteca. Es interesante destacar que no se ofrece en el $9 \%$ de las universidades públicas y en el 17\% de las privadas.

Se ofrece "asesoramiento sobre las revistas donde publicar" exclusivamente desde las bibliotecas en un 76,2\%, concretamente 24 bibliotecas del ámbito público y 8 del privado lo hacen. Además, el $6 \%$ de las públicas indican que lo realizan conjuntamente con otro servicio, mientras que este valor sube al $33 \%$ de las privadas. Es decir, es una prestación ofrecida de manera muy mayoritaria.

El servicio de "creación y mantenimiento de bibliografías" se ofrece en el 69\% de los casos desde la biblioteca, llegando al $73 \%$ en las bibliotecas de titularidad pública, pero solo al $58 \%$ en las privadas. Además destaca que el $14,3 \%$ de las instituciones lo ofrece desde cualquier otro órgano y el 16,7\% no lo presta. A pesar de considerar que es un servicio que fácilmente podría ser gestionado desde las bibliotecas, aún no encuentra una aceptación global. Mientras que la función de asesoramiento sobre metodologías de citación bibliográfica como se ha mencionado anteriormente se presta en la totalidad de las bibliotecas universitarias no ocurre lo mismo cuando se trata del mantenimiento de las bibliografías, aspecto que podría estar vinculado.

Respecto de la "elaboración de índices bibliométricos", 26 bibliotecas (62\%) los realizan, siendo en términos porcentuales el $57 \%$ en el caso de las públicas y el $75 \%$ en las privadas. Además es ofrecido conjuntamente por las bibliotecas de titularidad pública y otro estamento en 6 casos (19\%), mientras solo se consigna de manera conjunta en un caso en las universidades de titularidad privada. Sin llegar a ser una prestación ofrecida de manera total por las bibliotecas, sí que ocupa un papel destacado y en crecimiento cuando se efectúa conjuntamente con otro estamento. Puede considerarse que es un valor en alza y que ya forma parte del corpus principal de la cartera de servicios.

\subsubsection{Servicios prestados de manera residual}

Entre los servicios muy minoritarios, con poco nivel de implantación, encontramos la "gestión de comunicaciones entre el grupo", con un 9,5\% como servicio de biblioteca, solo 3 bibliotecas universitarias del ámbito público lo ofrecen y 1 en el privado. Es importante considerar que es mayor el número de instituciones que lo ofrecen conjuntamente a partir de otro estamento, situándose en alrededor del 17\% de los casos. Además se debe destacar que es un servicio que en el $30 \%$ de los casos no se ofrece.

Con menor implantación, la "preparación de borradores", solo se ofrece exclusivamente mediante la biblioteca en una universidad de titularidad pública. Cabe destacar que no se presta tal apoyo en 17 de las 30 instituciones públicas analizadas y en 5 de las 12 privadas. Es por tanto un servicio que podría extenderse en el que las bibliotecas podrían tener un papel destacado como en la gestión de las comunicaciones entre los grupos de investigación.

En este apartado, con un nivel bajo de respuesta, se encuentra también la "gestión de currículum" desde las bibliotecas universitarias. Sólo lo ofrecen ocho servicios de biblioteca, de las que siete son de universidades de titularidad pública. Cuando el servicio es ofrecido en las bibliotecas de universidades públicas, en el 16\% de los casos se realiza mediante la colaboración entre la biblioteca y otro estamento, y directamente desde otro servicio en un 19\%.

\subsubsection{Discusión de los resultados}

A tenor de los resultados presentados se pueden realizar las siguientes observaciones: en primer lugar, los servicios vinculados a la formación y asesoramiento en diferentes aspectos son los más implantados. Para su gestión/organización se precisa fundamentalmente un personal bibliotecario bien formado y con un importante nivel 
de actualización, pero no se precisa una inversión en infraestructuras o equipamientos. Responden al perfil más tradicional de la función bibliotecaria y por tanto más presente en las diferentes plantillas bibliotecarias.

Los servicios más novedosos, que están vinculados al nuevo rol bibliotecario van introduciéndose poco a poco. Tal vez las restricciones presupuestarias para la adquisición de tecnología, la modificación de espacios o los cambios de los perfiles profesionales, pueden ser las causas de este cierto "retraso" en su implantación.

Los servicios vinculados al nuevo rol bibliotecario de apoyo a la investigación van introduciéndose poco a poco. Tal vez las causas de este cierto "retraso" sean las restricciones presupuestarias para la adquisición de tecnología, la modificación de espacios o los cambios de los perfiles profesionales.

A modo general, se considera que las bibliotecas universitarias podrían mejorar y/o aumentar su catálogo de servicios considerando que tienen recursos y conocimientos para hacerlo. Para ello sería necesario que los responsables universitarios asuman que este es el servicio universitario adecuado para ofrecerlo.

\subsection{Modalidad de prestación de servicios de apoyo a la investigación}

El estudio incluía el análisis sobre la forma en la que se prestaban los servicios. En este sentido el 73,81\% de las bibliotecas afirman que combinan la forma presencial con la virtual (con atención telefónica). Resulta destacable que respecto de la posibilidad de que se usaran las redes sociales y los chats para ofrecer este asesoramiento, solo una universidad afirmara usarlas.

Así pues se observa que es posible una atención en diversas modalidades y que sería deseable, tal vez, diversificar y ampliar más los canales de comunicación usados incluyendo aquellos de mayor uso entre los usuarios como son las redes sociales.

\subsection{Formación del personal}

Un elemento clave para la prestación del apoyo a la investigación es disponer de personal bibliotecario bien formado, por ello el cuestionario incluía el análisis sobre los programas de formación continuada. Así se constata que casi la totalidad de las instituciones han realizado actividades formativas para sus trabajadores. En cuanto a las temáticas, un $95 \%$ han sido formados en gestores de referencias bibliográficas y de bases de datos, en la utilización de herramientas open access con un 90,5\%, y en menor medida, en el uso y en la importancia de índices de factores de impacto y en derechos de autor con un $85,7 \%$.

Por otro lado un 61,9 \% asistió a cursos de formación para la de elaboración de índices bibliométricos.

Mucha menos importancia ha tenido la formación a los empleados en herramientas y aplicaciones multimedia, que solo lo ha recibido un $45,2 \%$ de ellos.

Tomando en consideración la formación recibida por el personal de las bibliotecas, se puede afirmar que disponen de los conocimientos necesarios para ofrecer los servicios analizados.

\subsection{Implicación en la política de investigación}

Las bibliotecas como servicios de la universidad deben establecer sus actuaciones en consonancia con los objetivos institucionales. Por ello el cuestionario pretendía identificar de qué manera se implicaba específicamente en la política de investigación del centro y con los propios investigadores con la finalidad de ofrecer mayores posibilidades para el apoyo de los proyectos de investigación.

Se observa una dicotomía: mientras que en el 52,4\% de las universidades tiene una presencia específica en la definición de estas actuaciones (50\% en universidades públicas y 58\% en privadas); en un 40,5\% de ellas, esta es inexistente. El $31 \%$ de los servicios bibliotecarios participan en proyectos nacionales e internacionales de investigación, con temáticas variadas, tan solo un 4,8\% indican que participan en el proyecto Europeana Regia. Por lo tanto, es preocupante que casi una tercera parte de los servicios bibliotecarios no participen en proyectos nacionales e internacionales de investigación.

Por contra se ha constatado que el $88 \%$ tienen un papel activo en las iniciativas open access.

Además de la participación de la biblioteca en la política de investigación de la universidad, el contacto con los propios investigadores merece un comentario. En las universidades de titularidad pública se indica que se ha realizado 
un estudio previo de las necesidades del Personal Docente e Investigador (PDI) para crear servicios de apoyo a la investigación en un $53 \%$ de los casos, pero solo en un $41 \%$ en las privadas.

El $71,4 \%$ de los que han respondido indican que mantienen contacto con los grupos investigadores para conocer cuáles son sus necesidades. Entre estos el 76,7\% de titularidad pública, y el 58\% privada. El 21,3\% del total no mantiene habitualmente este contacto. Del total de servicios que mantienen contacto, el $60 \%$ de estos lo hace mediante conversaciones informales con investigadores. En menor medida se usa el correo electrónico como medio de comunicación.

Los aspectos vinculados al apoyo personalizado a los equipos de investigación no se prestan de manera significativa, pues no llegan al 50\% de las respuestas en el conjunto analizado, ni en el ámbito público, ni en el privado. Más de la mitad, un 64,3\%, de las bibliotecas disponen de datos para evaluar el uso de los servicios de apoyo a la investigación por parte del PDI. En el 71,4\% de los casos los servicios son gratuitos.

\section{CONCLUSIONES}

Las bibliotecas universitarias españolas miembros de REBIUN que han participado en el presente estudio, se comportan de manera diferente en lo concerniente a la prestación de servicios de apoyo a la investigación. Tampoco es constante que exista una tendencia clara en la prestación de los mismos si se trata de una institución de titularidad pública o privada. Además no parece que la bibliografía profesional más reciente, que como se ha visto previamente insiste en la necesidad de definir un servicio de apoyo a la investigación en las bibliotecas universitarias, haya impactado en la organización de los servicios de las bibliotecas. En este sentido el hecho de tener vínculos con REBIUN, red que elabora diferentes directrices y propuestas de actuación de servicios, como por ejemplo las contenidas en la línea estratégica II de su III Plan estratégico, haya necesariamente sido considerado.

El catálogo de servicios más comúnmente ofrecido se centra en la tarea formativa de la biblioteca en temas como el uso de las bases de datos especializadas, el funcionamiento de las citaciones bibliográficas y la información bibliográfica especializada. Además se consideran aspectos más actuales como la utilización de los repositorios documentales o las herramientas de open access. Le siguen otras prestaciones dentro del mismo ámbito, derivadas de la necesidad de los investigadores de difundir su producción científica y su evaluación, como el asesoramiento sobre las revistas donde publicar, la elaboración de índices bibliométricos y la creación y mantenimiento de bibliografías. En términos generales, la propuesta de ofrecer espacios de trabajo para los investigadores en la biblioteca recibe una buena respuesta, mientras que no lo hace el apoyo personalizado a los equipos de investigación.

En la misma línea, la combinación de la oferta de servicios presenciales y virtuales de apoyo a la investigación es la opción más extendida en las bibliotecas de las dos titularidades. Así mismo las bibliotecas de titularidad pública afirman tener datos que indican que el uso por parte del PDI de las bibliotecas es mayoritariamente virtual. Por otro lado se ha constatado que existen numerosos servicios que se ofrecen en colaboración de trabajo con otros servicios de sus instituciones para atender las necesidades de sus usuarios

Otro aspecto relevante es que los investigadores probablemente tienen necesidades de apoyo específicas según los proyectos que realizan. Por lo tanto, hay que incidir y mejorar el apoyo personalizado a los equipos de investigación atendiendo a que esta es una actividad clave de la universidad. Para ello las bibliotecas deben conocer bien este colectivo, saber adaptarse y especializarse para poder dar respuesta a sus necesidades.

Se recomienda que se efectúen estudios de necesidades informativas de los investigadores, o que se establezcan mecanismos, formales y ágiles, que permitan recopilar sistemáticamente esta información con el fin de ajustarse a sus expectativas. Además de colaborar más intensamente con los equipos de investigación, formarles es otro aspecto a potenciar.

Para prestar un buen servicio se debe apostar por la formación y actualización del personal bibliotecario en herramientas y en aquellos conocimientos que les permitan especializarse en los aspectos que requieren los investigadores y que pueden aportar un elemento de valor de la oferta de apoyo a la investigación de las bibliotecas universitarias. Existen elementos para considerar que puede realzarse mediante la formación del personal la prestación de servicios específicos que incidan en el apoyo a la investigación. Así pues el personal ha recibido formación en índices de medición de impacto y en creación de índices bibliométricos, y son estos aspectos que requieren de una mayor incidencia en el ámbito analizado para una prestación más extendida. 
De los datos analizados previamente se concluye que el apoyo a la investigación desde las bibliotecas de las universidades se presta de manera poco homogénea entre las universidades analizadas y debe responder a las necesidades específicas de cada una de las políticas de investigación de los propios centros. Así pues la biblioteca va tomando conciencia de su potencial en tanto que centro de apoyo a la investigación y va dirigiendo sus recursos y servicios para que sus prestaciones favorezcan el desarrollo de la investigación en la universidad. En este sentido es importante destacar que una parte muy significativa de las bibliotecas han considerado la necesidad de organizar prestaciones de apoyo a la investigación.

Para dar cumplimiento a la función investigadora las universidades tienen la necesidad de poner a disposición de los cuerpos docentes de sistemas de apoyo a la investigación. Estas deben analizar los recursos dispersos entre distintos servicios de la propia institución y plantearse concentrarlos en una única unidad, y la biblioteca es sin duda la mejor opción atendiendo al valor de sus aportaciones en este proceso. Esta podría ser una propuesta eficiente y efectiva, para la que a tenor de los resultados presentados, las bibliotecas están preparadas. Esta decisión implica potenciar e impulsar aún más esta especialización de apoyo y ofrecer una estructura con dotación de recursos, de mayor capacidad técnica y una integración efectiva en los estamentos universitarios que definen la política de investigación.

\section{NOTAS}

${ }^{1}$ Consejo Superior de Investigaciones Científicas. $<$ http://www.csic.es/ $>$.

${ }^{2}<$ http://www.rebiun.org/aprendizajeInvestigacion/Paginas/Objetivos-Generales-L\%C3\%ADnea-Estrat\%C3\%A9gica-2.aspx $>$.

3 REBIUN: < http://www.rebiun.org/documentos/Paginas/default.aspx >. Actualmente conformado por 50 universidades públicas, 26 universidades privadas y el CSIC.

\section{BIBLIOGRAFÍA}

CAMÓN LUIS, E.; REY MARTÍN, C. y BALAGUÉ MOLA, N. El suport a la recerca de les biblioteques universitàries catalanes: estat actual. BiD: textos universitaris de biblioteconomia i documentació, 2012, $\mathrm{n}^{\circ} 29$ (diciembre). $<$ http://bid.ub.edu/29/camon1.htm>. http://dx.doi.org/10.1344/BiD2012.29.6.

CARIDAD-SEBASTIÁN, M. y MARTÍNEZ-CARDAMA, S. El bibliotecario integrado en el aprendizaje universitario. El profesional de la información, 2013, marzo-abril, vol. 22, $\mathrm{n}^{\mathrm{o}} \quad 2, \quad \mathrm{p} . \quad 149-154$. http://dx.doi.org/10.3145/epi.2013.mar.09.

GALLO-LEÓN, J.P. La biblioteca es servicio y en ello está nuestro futuro. El profesional de la información, 2015 , vol. $24, n^{\circ} 2$, p. 87-93.

GANNON-LEARY, P.; BENT, M. y WEBB, Y. A destino oro en place of last resort? The research library of the future, its users and its Librarians. Library and information research, 2007, vol. 32, $\mathrm{n}^{\circ}$ 101, p. 3-14.

GARNER, I. Library support for research in a university contexto. 27th IATUL Conference. 2006, Oporto: IATUL.

IRIBARREN-MAESTRO, I. et al. Apoyando la investigación: nuevos roles en el servicio de bibliotecas de la Universidad de Navarra. El profesional de la información, 2015, vol. 24, nº 2, p. 131-137.

OCLC (2009). A Comparative review of research assessment regimes in five countries and the role of libraries in the research assessment process: piloto study Commissioned by OCLC Resarch (2009). Dublin: OCLC Research. $<$ http://www.oclc.org/resources/research/publications/library/2009/2009-09.pdf $>$. [Consulta: 25 de abril de 2017].

ROVIRA, A. Les biblioteques universitàries i el suport a la recerca. BiD: textos universitaris de biblioteconomia $i$ documentació, 2007, $\mathrm{n}^{\mathrm{o}} 18$ (junio). <http://bid.ub.edu/18rovir1.htm>. [Consulta: 26 de abril de 2017].

SHUMAKER, D. The embedded librarian: innovative strategies for taking knowledge where it's needed. New Jersey: Information Today, 2012.

SIPILA, S. Strong libraries, Strong Societies. El profesional de la información, 2015, vol.24, n 2, p. 95-101.

STOKKEM, J. E-research: access and support to university researchers. 29th IATUL Conference, 2008, Auckland: IATUL.

TORRES-SALINAS, D. Integrados en la investigación: los embedded librarians. Anuario ThinkEPI, 2011, vol. 5, p. 48-51. 